\title{
Lateral scattering potential of the PTCDA/Ag(111) interface state
}

\author{
A. Sabitova, ${ }^{1,2,3}$ R. Temirov, ${ }^{1,2, *}$ and F. S. Tautz ${ }^{1,2,3}$ \\ ${ }^{1}$ Peter Grünberg Institut (PGI-3), Forschungszentrum Jülich, 52425 Jülich, Germany \\ ${ }^{2}$ Jülich Aachen Research Alliance, Fundamentals of Future Information Technology, 52425 Jülich, Germany \\ ${ }^{3}$ Experimental Physics IV A, RWTH Aachen University, 52056 Aachen, Germany
}

(Received 23 March 2018; published 29 November 2018)

\begin{abstract}
A modified scheme of scanning tunneling spectroscopy data analysis has been used to reconstruct the band structure of a strongly dispersive two-dimensional unoccupied electronic state located at the commensurate interface between a monolayer of 3,4,9,10-perylene-tetracarboxylic dianhydride (PTCDA) and $\operatorname{Ag}(111)$ surface up to the fourth band in the reduced Brillouin zone of the molecular monolayer. The two-dimensional scattering potential, evaluated with the help of the measured band structure data, is highly corrugated. This shows that the accepted picture, describing the interface state of PTCDA/Ag(111) as an upshifted Shockley state of $\operatorname{Ag}(111)$, is incomplete. Our findings indicate that the hybridization of unoccupied molecular orbitals of PTCDA on one of the two sublattices with the metal states of $\operatorname{Ag}(111)$ substantially influences the interface state properties.
\end{abstract}

DOI: 10.1103/PhysRevB.98.205429

\section{INTRODUCTION}

The atomic-scale patterning [1,2] of interfaces consisting of large organic molecules and metal surfaces allows the crafting of structures with novel electronic properties and, ultimately, designed quantum states [3-5]. To take full advantage of this approach to quantum state engineering, it is vital to develop a detailed understanding of such moleculemetal interfaces in their native, i.e., unstructured, form. In this context, dispersive interface states which often form at these interfaces [6-13] are highly relevant because these are states that are most susceptible to the effects of lateral structuring.

The interface of 3,4,9,10-perylene-tetracarboxylic dianhydride (PTCDA) with the $\operatorname{Ag}(111)$ surface was the first molecule-metal interface to reveal an unoccupied, parabolically dispersive two-dimensional (2D) interface state [6]. Initially characterized as a hybrid molecule-metal state and attributed to a metal-mediated coupling between adjacent unoccupied molecular orbitals [6], it has been further scrutinized by two-photon photoemission (2PPE) experiments [9] and density functional theory (DFT) calculations $[14,15]$. Based on its effective mass $m^{*} \approx 0.5 m_{\mathrm{e}}[6,9]$, relatively short lifetimes [9], and a strong wave function overlap with the substrate $[14,15]$, it was concluded that the interface state is closely related to the Shockley state of bare $\operatorname{Ag}(111)$, shifted up in energy by the adsorption of the molecular layer [12,13,15-17], in close analogy to earlier reports on such weakly interacting interfaces as $\mathrm{Xe} / \mathrm{Cu}(111)$ [18] and $\mathrm{NaCl} / \mathrm{Cu}(111)$ [19] and some weakly adsorbed molecular layers [17,20,21].

Based on this finding, Armbrust et al. have shown that an analytical one-dimensional (1D) model potential describes the interface state onset energy $E_{0}$ based on only the vertical adsorption distance $d$ [22]. Within this model, the average

*Corresponding author: r.temirov@fz-juelich.de carbon plane of the adsorbate modifies the $1 \mathrm{D}$ barrier at the metal surface, and wave function matching at this modified barrier selects the energy of the interface state [23]. This yields the upshift of the interface state energy with respect to the surface state energy of the clean metal that is universally observed for experimental adsorption distances [22].

It is clear, however, that this 1D model cannot capture several important aspects of the actual interface state. First, a possible scattering of electrons in the interface state by the periodic lattice of the molecular layer is neglected. Second, the influence of the internal molecular structure is not accounted for. Yet it is known that molecular distortions influence the overlap of the interface state with the metal and thus its lifetime [12]. Third, the possibility that unoccupied molecular orbitals may contribute to the interface state, as originally proposed in Ref. [6], thus forming a true hybrid molecule-metal state, is precluded. We note in this context that the electronic properties of at least some strongly hybridized molecular-metal interfaces $[10,11,22]$ cannot be rationalized with the 1D model. Finally, the 1D model cannot possibly describe the interface state in and around laterally patterned nanostructures because by construction it is restricted to the vicinity of the $\vec{k}=0$ point [22] (note that throughout the paper $\vec{r}$ and $\vec{k}$ refer to lateral position and wave vectors).

An experimental approach to resolve these issues is measuring the lateral band structure of the interface state over the complete first Brillouin zone (1BZ) because the band gaps at the $1 \mathrm{BZ}$ boundaries embody information on the scattering of interface state electrons, and this scattering in turn will be determined by the molecular lattice as such, possible molecular distortions, and, if present, any hybridization of molecular orbitals with the free-electron-like interface state. From the gaps, one can, in principle, determine the lateral scattering potential since the former are determined by the latter's Fourier components.

Although the PTCDA/Ag(111) interface is a well-known prototypical example of a molecule-metal interface of the 
type of interest here [24], the complete band structure of its interface state is not known because its measurement is challenging. The unoccupied character of the interface state rules out photoemission studies [25]. While 2PPE spectroscopy can address unoccupied states in principle, the abundance of rotational domains in the present system leaves $2 \mathrm{PPE}$ data $[9,12,13]$ difficult to interpret. Scanning tunneling spectroscopy (STS) is thus one of the few suitable techniques. However, STS has its own principal problems in providing band maps of electronic states close to the $1 \mathrm{BZ}$ boundaries. These stem from the superposition of topographic and electronic contrasts, as detailed below. Here, we circumvent this problem by a modified STS data analysis approach and derive the band structure of the PTCDA/Ag(111) interface state across the whole $1 \mathrm{BZ}$ up to the fourth band. On the basis of these data, we find a surprisingly strong lateral scattering of the interface state by the molecular layer.

\section{EXPERIMENTAL DETAILS}

The experiments were conducted in a commercial CREATEC scanning tunneling microscope (STM) operating in ultrahigh vacuum and at a base temperature of $5 \mathrm{~K}$. The $\operatorname{Ag}(111)$ crystal was prepared in situ by standard surface preparation techniques. PTCDA molecules were evaporated from a home-built Knudsen cell. Submonolayer coverages of $20 \%-50 \%$ of a monolayer were employed. The STM tip, cut with a focused ion beam from a $20-\mu \mathrm{m}$ PtIr wire, was cleaned by repeated cycles of applying voltage pulses and careful indentations into the clean $\mathrm{Ag}(111)$ surface. In its final state the tip is most likely fully covered by silver atoms. The sharpness and the cleanness of the tip were controlled by performing STS of the $\mathrm{Ag}(111)$ Shockley state. STS was performed using lock-in detection with modulation amplitudes of $4-10 \mathrm{mV}$ and modulation frequencies of $0.6-6 \mathrm{kHz}$.

As pointed out in the Introduction, the results presented here crucially depend on the application of a modified STS data analysis scheme: feature detection (FD) scanning tunneling spectroscopy. Here, FD STS involves recording an array of $d I / d V(V)$ spectra on a $64 \times 64$ grid of $(x, y)$ points above a $50 \times 50 \AA^{2}$ defect-free area of PTCDA/Ag(111). Since each spectrum takes approximately $15 \mathrm{~s}$ to record, the grid is scanned in constant-current mode, as this limits the influence of vertical drift and thus permits long acquisition times ( $\simeq 18 \mathrm{~h}$ ). A custom algorithm is then used to detect spectral features in every $d I / d V$ spectrum. Although the types of the detected features may include peaks, shoulders, steps, etc., for simplicity we describe here a routine that detects only $d I / d V$ peaks. They are detected by smoothing the $d I / d V$ spectra, calculating $d^{2} I / d V^{2}$, and registering every $e V_{\mathrm{b}}$ at which $d^{2} I / d V^{2}$ crosses through zero from positive values as the energy of a peak [Figs. 1(a) and 1(b)]. Each of the so-detected peaks is assigned a weight $W$, defined as the height of the corresponding $d^{2} I / d V^{2}$ curve segment from maximum to minimum [Fig. 1(b)]. Finally, all detected peaks are compiled into a histogram to which every peak contributes $W$ at its energy $e V_{\mathrm{b}}$. This energy distribution histogram (EDH) is the principal result of FD STS, from which we obtain here the lateral band structure of the PTCDA/Ag(111) interface state.

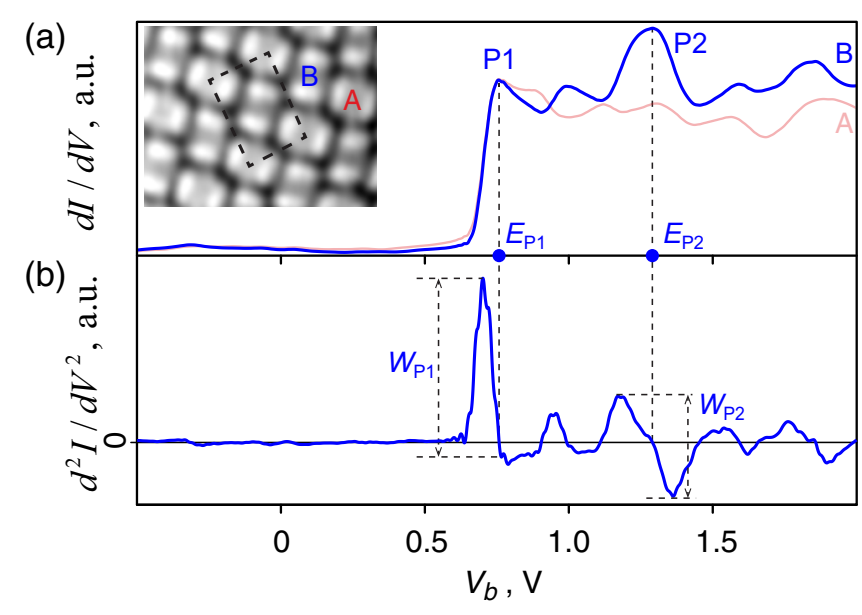

FIG. 1. Structural and electronic properties of PTCDA/Ag(111). (a) STS spectra (smoothed), measured above the centers of type-A (red) and type-B (blue) PTCDA molecules on $\operatorname{Ag}(111)$. The inset shows a constant-current STM image of PTCDA/Ag(111), measured at $V_{\mathrm{b}}=-340 \mathrm{mV}$. The unit cell with $\left|\vec{a}_{1}\right|=18.96 \AA$ and $\left|\vec{a}_{2}\right|=$ $12.61 \AA$ is indicated with a black dashed line. Type-A molecules, occupying the corners of the drawn unit cell, are aligned with the [1 10$]$ direction of the substrate [26]. (b) Numerical derivative $d^{2} I / d V^{2}$, obtained from the smoothed STS spectrum in (a), as used for the detection of $d I / d V$ peaks (type-B molecule). Dashed lines indicate two examples of detected peaks, labeled P1 and P2. $E_{\mathrm{P} 1}$ and $E_{\mathrm{P} 2}$ denote the detected peak positions; $W_{\mathrm{P} 1}$ and $W_{\mathrm{P} 2}$ are the corresponding weights.

\section{RESULTS}

We start our analysis with Fig. 1(a), which recalls the STS signature of the interface state: the characteristic steplike feature at $E_{0}=e V_{\mathrm{b}}=700 \mathrm{meV}$ which marks the onset of the unoccupied 2D interface state band [6]. Next, we apply the well-known technique of Fourier transform STS (FT STS) $[27,28]$ to obtain the dispersion of the interface state in the vicinity of the $\bar{\Gamma}$ point; this will also illustrate the limitations of STS as applied to the study of dispersive states.

FT STS maps the dispersion by recording a set of constantcurrent $d I / d V$ images, each at a fixed voltage $V_{\mathrm{b}}$. In the case of PTCDA/Ag(111), $V_{\mathrm{b}}$ in the range from 0.6 to $2 \mathrm{~V}$ are selected. Strong scattering of the interface state by defects in the PTCDA monolayer produces $V_{\mathrm{b}}$-dependent patterns, the well-known (energy-resolved) Friedel oscillations. Generally speaking, Friedel oscillations are stationary modulations in the charge density that originate from the screening of the defects by metal electrons. Note that in the present case the Friedel oscillations originate from electrons injected into unoccupied states at the PTCDA/Ag(111) interface. Figure 2(a) displays an example image at a fixed bias voltage of $930 \mathrm{mV}$. Due to the predominance of the Friedel oscillations in these images, their fast Fourier transforms (FFT) yield contours in $k$ space which define the location of screening states with the corresponding energy [Fig. 2(b)]. Plotting the contours as a function of the energy, the dispersion of the screening states can be reconstructed [Fig. 2(c)]. In doing so, one must, however, keep in mind that modulations in images such as Fig. 2(a) occur in the charge density which is proportional 
(a)

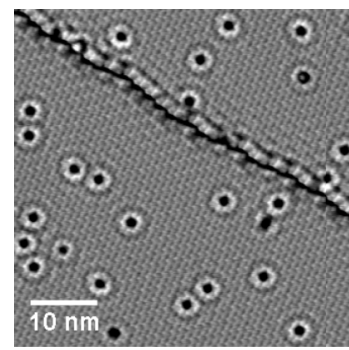

(b)

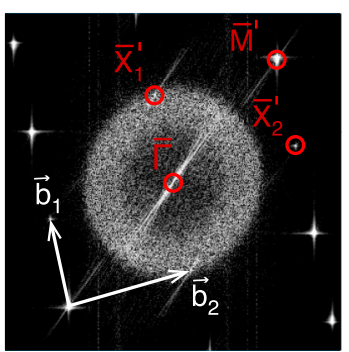

(c)

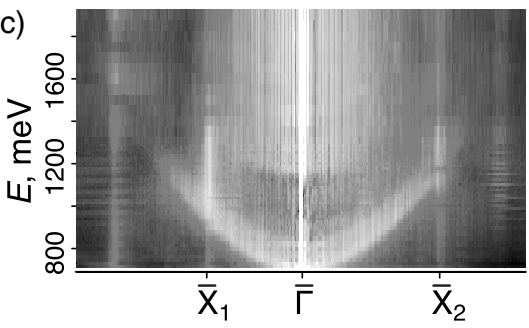

(d)

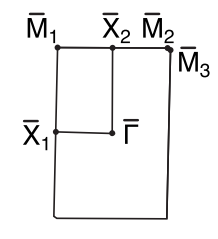

FIG. 2. PTCDA/Ag(111) interface state dispersion as determined by FT STS. (a) Constant-current $d I / d V$ image measured at $V_{\mathrm{b}}=930 \mathrm{mV}$. (b) FFT of the image shown in (a). White arrows: reciprocal lattice vectors of the PTCDA/Ag(111) interface. When the ringlike FFT pattern reaches the red circles labeled $\overline{\mathrm{X}}_{1}^{\prime}, \overline{\mathrm{X}}_{2}^{\prime}$, and $\overline{\mathrm{M}}^{\prime}, \vec{k}$ hits the $1 \mathrm{BZ}$ boundaries at the corresponding $\bar{X}_{1}, \bar{X}_{2}$, and $\bar{M}$ points. Note that $\bar{X}_{1}^{\prime}, \bar{X}_{2}^{\prime}$, and $\bar{M}^{\prime}$ are not located at the 1BZ boundary (see text). (c) Parabolic dispersion of the interface state extracted from FT STS. The horizontal $\vec{k}$ scale is calibrated using FFT spots of the molecular lattice which correspond to the reciprocal vectors $\left|\vec{b}_{1}\right|=2 \pi /\left|\vec{a}_{1}\right|$ and $\left|\vec{b}_{2}\right|=2 \pi /\left|\vec{a}_{2}\right|$ shown in (b). (d) First Brillouin zone of PTCDA/Ag(111). The truncated corners in the top right and bottom left result from the slightly skewed (approximately $1^{\circ}$ ) real-space unit cell of PTCDA/Ag(111) [24]. For simplicity, this tiny deviation from a rectangular $1 \mathrm{BZ}$ is neglected.

to the square of the wave function (in STM only the square of the wave function can be imaged). Therefore, the radius of the ringlike FFT pattern in Fig. 2(b) is equal to $2|\vec{k}|$, where $\vec{k}$ is the wave vector of the wave function underlying the corresponding energy-resolved Friedel oscillation [27]. As a consequence, $\vec{k}$ reaches the $1 \mathrm{BZ}$ boundaries when the FFT ring with a radius of $2|\vec{k}|$ crosses the reciprocal lattice points [indicated in red in Fig. 2(b)].

Figure 2(c), obtained from the stack of FT STS images in the relevant energy range, shows that the interface state has a parabolic dispersion with an effective mass of $m^{*} \approx$ $0.48 m_{e}$, agreeing well with previously published data $[6,9]$. But Fig. 2(c) also shows vertical bars of strong intensity at the $1 \mathrm{BZ}$ boundaries $\bar{X}_{1}$ and $\bar{X}_{2}$. These stem from reciprocal lattice vectors which appear in the STM images irrespective of energy since they are of structural origin. This reveals a severe limitation of FT STS: in the most interesting region where band gaps may occur, the electronic band structure as derived from FT STS is masked by reciprocal lattice points, such that band gaps cannot be determined.

This problem is, in fact, generic because in STS $d I / d V$ images with their lateral variations, recorded either at constant current or constant height, are interpreted as maps of the laterally varying local density of states (LDOS). This approach works well on atomically flat surfaces but fails as soon as a significant corrugation is present because the corrugation of the surface also causes variations of $d I / d V$ that are superposed on the LDOS signal [29], regardless of whether the constant current or constant height modes are employed. Because the pure topography is inaccessible and even illdefined at the atomic scale, a disentanglement of topography and LDOS is difficult, apart from special cases: For example, if the characteristic length scales (periodicities) of the LDOS pattern and the surface corrugation are well separated, the superposition problem is not so severe [19]. However, the LDOS variation due to the interface state band at the $1 \mathrm{BZ}$ boundaries coincides precisely with the periodic corrugation of the PTCDA/Ag(111) monolayer, which makes the correct interpretation of the STS data at the $1 \mathrm{BZ}$ boundaries very difficult.

To circumvent this problem, we apply FD STS, as described in the previous section, to study the band structure of the PTCDA/Ag(111) interface state. Since we are particularly interested in the band gaps at the $1 \mathrm{BZ}$ boundaries, we use a defect-free image in which we expect only Bragg scattering (restricted to wave vectors close to the $1 \mathrm{BZ}$ boundaries) and no impurity scattering. The EDH extracted from $d I / d V$ data on PTCDA/Ag(111) is shown in Fig. 3(h). It represents the weighted distribution of all detected $d I / d V$ peaks on the energy axis (see above). The precise meaning of the EDH will become apparent once we analyze the spatial distribution of the $d I / d V$ peaks that contribute to a specific histogram feature. First, we scrutinize the relation of the EDH to the band structure of the interface state in as far as the latter is known, e.g., from FT STS.

Figure 3(h) shows that the lowest EDH feature corresponds to the bottom of the parabolic interface state band at the $\bar{\Gamma}$ point. Note that the shift of $\approx 50 \mathrm{meV}$ between the bottom of the band and the corresponding EDH feature occurs because the algorithm described here detects peaks, while the interface state onset energy $E_{0}$ is defined by the energy at which the $d I / d V$ step [30] [see Figs. 1(a) and 1(b)] reaches half its amplitude. Figure 3(h) also shows that EDH features a and b bracket the energy at which the free-electron dispersion parabola from Fig. 2(c) crosses the $\bar{X}_{1}$ point of the $1 \mathrm{BZ}$. Similarly, features c and d bracket the parabolic dispersion at $\bar{X}_{2}$. This suggests that the EDH features may mark energies at which the density of states of the PTCDA/Ag(111) interface state is high due to the flattening of the bands close to the $1 \mathrm{BZ}$ boundaries.

To validate this conjecture, we analyze the spatial distribution of the EDH features on the $(x, y)$ grid on which $d I / d V$ spectra were recorded. To this end, all pixels that contribute to a specific EDH feature by more than a chosen threshold value $W_{0}$ are assigned a value of 1 , while all other pixels remain 0 . The resulting feature distribution maps (FDMs), in contrast to the traditional STS images, do not map the intensity of the $d I / d V$ signal at a particular energy but rather reveal the area where a chosen EDH feature has been detected. This eliminates the contribution of the surface topography, leaving behind a highly posterized image of the LDOS, as we will recognize when turning to the thus-generated FDM of the PTCDA/Ag(111) interface state.

Inspecting the FDM of features $a$ and $b$ [left panels of Figs. 3(a) and 3(b)], we observe two stripe patterns, each 

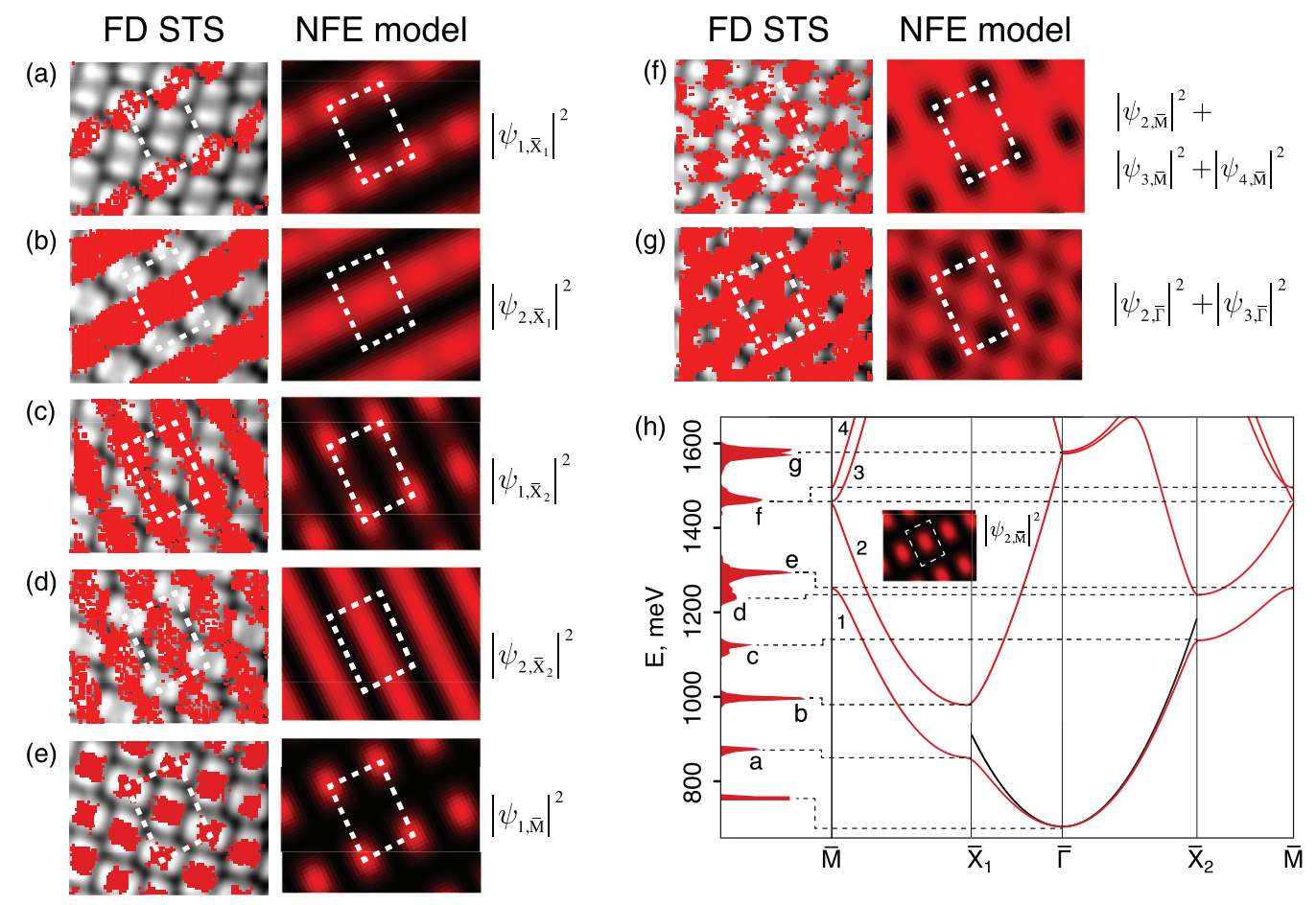

FIG. 3. Band structure of the PTCDA/Ag(111) interface state as derived from FD STS and a NFE model calculation fitted to FD STS data. (a)-(g) Left column: Experimental feature distribution maps (FDMs) extracted from specific features of the measured energy distribution histogram (EDH) displayed on the left-hand side of (h). Right column: $\left|\psi(\vec{r})_{i, \vec{k}}\right|^{2}$ of the calculated NFE band structure for (a) and (b) $\vec{k}=\bar{X}_{1}$, (c) and (d) $\bar{X}_{2}$, (e) and (f) $\bar{M}$, and (g) $\bar{\Gamma}$ points of PTCDA/Ag(111), based on the approximated rectangular 1BZ. The real-space unit cell defined in the inset of Fig. 1(a) is indicated by white dashed rectangles. (h) Experimental EDH (left) and 2D NFE model band structure (right), the latter calculated with $U_{ \pm \vec{b}_{1}}=38 \mathrm{mV}, U_{ \pm \vec{b}_{2}}=57 \mathrm{mV}, U_{ \pm\left(\vec{b}_{1}+\vec{b}_{2}\right)}=U_{ \pm\left(\vec{b}_{1}-\vec{b}_{2}\right)}=-61 \mathrm{mV}$, as obtained by fitting the NFE model to the EDH (see text). Only the four lowest bands of the calculated band structure are shown. The black parabola shows the dispersion of a free-electron state with $m^{*}=0.48 m_{e}$ and an onset energy of $700 \mathrm{meV}$. Horizontal dashed lines indicate the correspondence between the features of the model band structure and EDH features. The inset shows the calculated $\left|\psi(\vec{r})_{2, \overrightarrow{\mathrm{M}}}\right|^{2}$, which has no counterpart in the experimental EDH and FDM (see text).

with the periodicity of the molecular lattice but laterally offset against each other by half a lattice constant. This is exactly what is expected from the Bragg scattering of nearly free electrons in the periodic lattice of the PTCDA molecules. Bragg scattering occurs for wave vectors at the $1 \mathrm{BZ}$ boundaries, i.e., for wavelengths twice the lattice constant, but similar to FT STS detection of Friedel oscillations discussed above, FD STS is also sensitive to the square of the wave function; hence, we observe charge density modulations with the periodicity of the lattice. Evidently, the strong posterization does not pose any problem in identifying sinusoidal charge density modulations.

It is well known that Bragg-scattered standing waves come in phase-shifted pairs, with one wave lowered in energy in comparison to the traveling free electron (without scattering), and the other raised in energy [23]. This is exactly what we observe in Fig. 3(h) for EDH features a and $b$ and also for features $\mathrm{c}$ and $\mathrm{d}$. Therefore, we conclude that EDH features a, $\mathrm{b}, \mathrm{c}$, and d reveal the edges of the band gaps that arise from the Bragg scattering of the interface state, in analogy with a density of states that is high due to the flatness of the bands at the 1BZ boundaries. The local density of states is given by the corresponding FDM, as Figs. 3(a)-3(d) show.

We employ the band gap information provided by the $\mathrm{EDH}$ for the reconstruction of the full band structure of the PTCDA/Ag(111) interface state. From the Schrödinger equation

$$
\left[-\frac{\hbar^{2}}{2 m} \nabla^{2}+U(\vec{r})\right] \psi(\vec{r})=\epsilon \psi(\vec{r}),
$$

with a (scattering) potential $U(\vec{r})=\sum_{\vec{G}} U_{\vec{G}} e^{i \vec{G} \cdot \vec{r}}$, we calculate a model band structure of a rectangular lattice in the nearly-free-electron (NFE) approximation [23]. Because the real-space unit cell of PTCDA/ $\operatorname{Ag}(111)$ is slightly skewed [26], its $1 \mathrm{BZ}$ is not rectangular but has two truncated corners and thus three inequivalent $\bar{M}$ points [Fig. 2(d)]. Here, we neglect this tiny deviation for simplicity and assume a rectangular 1BZ. Using a minimal set of eight nonzero Fourier components $\left(U_{ \pm \vec{b}_{1}}, U_{ \pm \vec{b}_{2}}, U_{ \pm\left(\vec{b}_{1}+\vec{b}_{2}\right)}, U_{ \pm\left(\vec{b}_{1}-\vec{b}_{2}\right)}\right)$ that encode the structure of a rectangular lattice with two inequivalent sites per unit cell, we treat them as four fit parameters $\left(U_{+\vec{b}_{1}}=\right.$ $U_{-\vec{b}_{1}}$, etc., for symmetry reasons) in order to fit the $\bar{X}_{1}$ and $\bar{X}_{2}$ band gap energies to EDH features a, b, c, and d. The resultant NFE band structure is displayed in Fig. 3(h). We also plot the calculated wave functions' square moduli $\left|\psi(\vec{r})_{i, \vec{k}}\right|^{2}$ of the four lowest-energy bands, $i=1, \ldots 4$, at $\vec{k}=\bar{X}_{1}, \bar{X}_{2}, \bar{M}$, and $\bar{\Gamma}$ in the $1 \mathrm{BZ}$ and place these plots next to the corresponding FDM [Figs. 3(a)-3(g)]. 


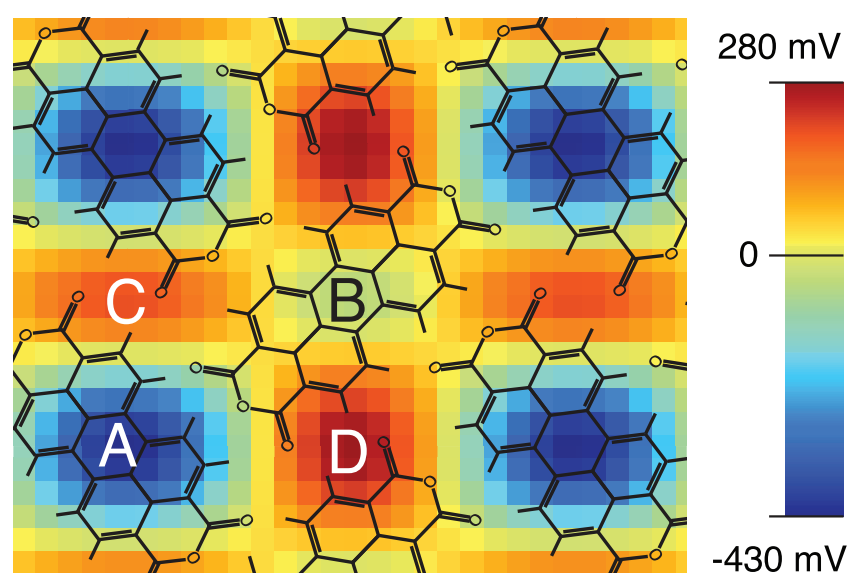

FIG. 4. Map of 2D scattering potential $U(\vec{r})$ of the PTCDA/Ag(111) interface state, overlaid with the structure of the PTCDA monolayer. The corners of the PTCDA/Ag(111) unit cell are occupied by type-A molecules; the centers are occupied by type-B molecules [see the inset of Fig. 1(a)]. Values of $U(\vec{r})$ at high-symmetry points of the unit cell are $U(\mathrm{~A})=-420 \mathrm{meV}$, $U(\mathrm{~B})=-50 \mathrm{meV}, U(\mathrm{C})=190 \mathrm{meV}, U(\mathrm{D})=260 \mathrm{meV}$.

We observe that $\left|\psi(\vec{r})_{1, \bar{X}_{1}}\right|^{2},\left|\psi(\vec{r})_{2, \overline{\mathrm{X}}_{1}}\right|^{2},\left|\psi(\vec{r})_{1, \overline{\mathrm{X}}_{2}}\right|^{2}$, and $\left|\psi(\vec{r})_{2, \bar{X}_{2}}\right|^{2}$ correspond precisely to the FDMs of EDH features a, b, c, and d [Figs. 3(a)-3(d)]. Remarkably, the FDMs of features $e, f$, and $g$, whose energies were not directly fitted by the NFE model, also display a very good correspondence to the features of the model band structure: The FDM of feature g corresponds to $\left|\psi(\vec{r})_{2, \bar{\Gamma}}\right|^{2}+\left|\psi(\vec{r})_{3, \bar{\Gamma}}\right|^{2}$ [Fig. 3(g)], feature f contains $\left|\psi(\vec{r})_{3, \overline{\mathrm{M}}}\right|^{2}+\left|\psi(\vec{r})_{4, \overline{\mathrm{M}}}\right|^{2}[$ Fig. 3(f) and inset in Fig. 3(h)], and feature e contains $\left|\psi(\vec{r})_{1, \overline{\mathrm{M}}}\right|^{2}$ [Fig. 3(e)]. Only $\left|\psi(\vec{r})_{2, \overline{\mathrm{M}}}\right|^{2}$, plotted in the inset in Fig. 3(h), does not find a counterpart in the FD STS data. Note, however, that the $i=2$ band does not flatten substantially at $\bar{M}$ and hence may not produce a sufficient LDOS increase to be resolved with FD STS. We remark that we see in Fig. 3(e) a pattern of apparent similarity to $\left|\psi(\vec{r})_{2, \overline{\mathrm{M}}}\right|^{2}$. However, this does not appear at the predicted energy and therefore must have a different origin, which we will discuss below.

Once the band structure of the interface state has been established, the potential $U(\vec{r})$ is straightforwardly evaluated from the sum of the fitted Fourier components $U_{\vec{G}}$. Figure 4 reveals that $U(\vec{r})$ is strongly corrugated, extending $420 \mathrm{meV}$ below and $260 \mathrm{meV}$ above the onset of the interface state band at $700 \mathrm{meV}$. The properties of $U(\vec{r})$ can be summarized as follows (Fig. 4): (i) the centers of both type-A and type-B molecules are attractive $[U(\mathrm{~A}), U(\mathrm{~B})<0]$, (ii) the centers of type-B molecules are considerably less attractive than the centers of type-A molecules $[U(\mathrm{~A})<U(\mathrm{~B})]$, and (iii) the carboxylic oxygen atoms on both type-A and type-B molecules are strongly repulsive towards electrons in the interface state $[U(\mathrm{C}), U(\mathrm{D})>0]$. In the next section we discuss the implications of these findings.

\section{DISCUSSION}

According to the 1D model of the interface state [22], the vertical adsorption distance $d$ of the molecule above the metal surface is a sensitive control parameter that determines the interface state energy $E_{0}$ at $\vec{k}=0$. Assuming that the $E_{0}(d)$ curve which describes the relation between the vertical adsorption height and the interface state energy in the 1D model also predicts the lateral variation, due to vertical molecular distortions, of the potential in which the free-electron-like interface state electrons move, the $0.18-\AA$ bending of carboxylic oxygen atoms of PTCDA towards $\mathrm{Ag}(111)$ [31] may be expected to yield $U(\vec{r}) \simeq 0.3 \mathrm{eV}$ at the lateral positions of the carboxylic oxygens, under the assumption that a strictly planar molecular layer would give $U \simeq 0$. In good agreement with this prediction, our data indeed reveal that the potential of interface state electrons in regions $\mathrm{C}$ and $\mathrm{D}$, which contain the carboxylic oxygens atoms, is increased by $0.26 \mathrm{eV}$ with respect to the onset energy of the interface state (Fig. 4). However, neither the attractive potential in regions $\mathrm{A}$ and $\mathrm{B}$ nor the difference between $U(A)$ and $U(B)$ can be explained by adsorption height variations. For example, in order to account for the different attractivenesses of type-A and -B molecules by their different adsorption heights using the $E_{0}(d)$ curve of Ref. [22], type-B molecules would have to be $0.2 \AA$ closer to the surface than type-A molecules to increase the potential at their positions by $0.37 \mathrm{eV}$. This prediction disagrees with recent measurements that find type-B molecules only $0.05 \AA$ closer to the surface than type-A molecules [32]. We therefore need to search for other mechanisms that explain the findings $U(A), U(B)<0$ and $U(A)<U(B)$.

In addition to the adsorption height, at least two additional factors may influence the corrugation of $U(\vec{r})$. These are electrostatic fields within the molecular layer due to an inhomogeneous charge distribution and the possible hybridization of unoccupied molecular orbitals $[6,14]$ with the interface state as derived in the 1D model [22].

The in-plane quadrupole moment of PTCDA is expected to lower the potential in the centers of both type-A and -B molecules and raise it at their dianhydride groups [33]. While this leads to $U(\mathrm{~A}), U(\mathrm{~B})<0$, this effect is not strong enough for the given charge distribution inside the molecule. Moreover, the quadrupole moment does not explain the pronounced difference between $U(\mathrm{~A})$ and $U(\mathrm{~B})$ [34]. This difference could, in principle, result from the charge transfer across the interface from $\mathrm{Ag}(111)$ into PTCDA. Indeed, we recently found that type-B molecules adsorb slightly closer to the surface [32], and hence, they should receive more negative charge from $\operatorname{Ag}(111)$ [26], which would raise $U(\mathrm{~B})$ with respect to $U(\mathrm{~A})$. The small amount of the total transferred charge $(\sim 0.35 e$ [26]), however, makes it unlikely that the observed difference of $370 \mathrm{meV}$ between $U(\mathrm{~A})$ and $U(\mathrm{~B})$ can be explained exclusively by this mechanism.

Finally, we discuss the possibility of a hybridization of molecular orbitals with the free-electron-like interface state. Before analyzing our data in this respect, we briefly recall the treatment of the molecular layer in the 1D model [22]. In this model, $E_{0}$ is obtained by solving the Schrödinger equation in a potential that is the sum of model potentials for the metal surface and the organic monolayer. The former is taken from Ref. [35], while the latter is adapted from freestanding graphene in the context of modeling its image potential states [36]. The image potential states of graphene exist in a wide 
band gap of its electronic structure [36]. By using the model potential obtained from graphene in the present case of the PTCDA/Ag(111) interface, the 1D model implicitly assumes the presence of a large gap in the electronic structure of the molecular monolayer as well. This assumption, however, is not correct since both the lowest unoccupied molecular orbital $(\mathrm{LUMO})+1$ and the LUMO +2 of PTCDA are found very close to $E_{0}[6,14,26]$.

The presence of unoccupied orbitals of PTCDA in the energy range of the free-electron-like interface state offers the principal possibility that these orbitals could hybridize with the latter. In order to demonstrate that such a hybridization is, in fact, supported by the experimental data, we return to the discussion of the discrepancy between the experimental FDM images and the NFE wave functions at the $\bar{M}$ point (Fig. 3): While the FDM of feature e, which, according to the NFE band structure in Fig. 3(h), should be the map of $\left|\psi_{1, \bar{M}}\right|^{2}$ [Fig. 3(e)], has an excess spot located on sublattice $\mathrm{B}$, precisely this spot is missing in the FDM of feature f, which, according to Fig. 3(h), should correspond to the sum $\left|\psi_{2, \overline{\mathrm{M}}}\right|^{2}+\left|\psi_{3, \overline{\mathrm{M}}}\right|^{2}+\left|\psi_{4, \overline{\mathrm{M}}}\right|^{2}$ [Fig. 3(f)]. Naively, one may assume that the problem arises because the energy of $\psi_{2, \overline{\mathrm{M}}}$ is overestimated in the NFE model and that the discrepancy could be eliminated by tuning this energy closer to $\psi_{1, \overline{\mathrm{M}}}$ or even by reversing the energy ordering of $\psi_{1, \overline{\mathrm{M}}}$ and $\psi_{2, \overline{\mathrm{M}}}$. Such a correction, however, is not possible, as it would also rescale (or even invert) the $\bar{X}_{1}$ and $\bar{X}_{2}$ gaps, thus destroying the excellent agreement between the experimental FDM and NFE model in Figs. 3(a)-3(d). Therefore, we are led to the conclusion that the feature located on the type-B molecule in Fig. 3(e) cannot belong to the dispersive band state.

An indication that the excess feature in Fig. 3(e) belongs to a nondispersive molecular resonance of type-B molecules can, in fact, be spotted in the experimental data: The $d I / d V$ spectra of type-A and type-B molecules in Fig. 1(a) show that, in contrast to type A, type-B molecules exhibit a wellpronounced $d I / d V$ peak $\mathrm{P} 2$ at approximately $1300 \mathrm{meV}$. Although type-A molecules also have a $d I / d V$ peak at a similar energy, the much higher intensity of P2 indicates its special character. If we recall that the unavoidable averaging over $\vec{k}$ tends to smear out all dispersive $d I / d V$ features in STS experiments, we may indeed ascribe $\mathrm{P} 2$ to a nondispersive state localized exclusively on type-B molecules. It is this state that gives rise to the excess spot in Fig. 3(e).

Two questions then immediately arise: What is the nature of this localized, nondispersive state, and why does such a state not exist on type-A molecules? Regarding the first question, we note that the position of $\mathrm{P} 2$ corresponds well to the energies of the LUMO $+1 / 2$ as predicted by DFT calculations [26]. Regarding the second question, we propose that the LUMO+2 on type-A molecules hybridizes strongly with the free-electron-like interface state and thus becomes an indistinguishable part of the band structure in Fig. 3(h). Considering a possible hybridization of the LUMO+2 with the free-electron-like interface state (due to its symmetry, the LUMO+1 cannot couple [26]), we remark that such a hybridization should be most effective at the $\bar{M}$ point because there the wave functions of the free-electron-like state are Bragg scattered into standing waves that are concentrated on PTCDA molecules. The disparity of the coupling of the $\mathrm{LUMO}+2$ on the two sublattices to the free-electron-like interface state is explained by a slight difference in the initial energies of the two LUMO+2. Specifically, DFT calculations find the LUMO +2 of type-B molecules $\phi_{\mathrm{B}} \sim 110 \mathrm{meV}$ below that of type $\mathrm{A} \phi_{\mathrm{A}}$ [26]. The origin of this difference is a slightly stronger charge transfer into type-B molecules that is well known from the relative shifts of the highest occupied molecular orbital and LUMO observed in STS measurements [6,26].

Taking all the evidence together, the following scenario emerges: Bragg scattering of the free-electron-like interface state at $\bar{M}$ concentrates wave functions at either of the two sublattices, leading to the initial opening of a gap at $\bar{M}$. Because of stronger electrostatic repulsion from the larger charge transferred into type-B molecules, the standing wave function on sublattice B appears above the incipient gap and thus becomes $\psi_{2, \overline{\mathrm{M}}}$, while the standing wave below the gap $\psi_{1, \overline{\mathrm{M}}}$ is concentrated on sublattice A. Equivalently, this implies $U(A) \lesssim U(B)$. The initial gap opening via electrostatics is amplified by the hybridization: Being better aligned, molecular orbital $\phi_{\mathrm{A}}$ on sublattice A can effectively hybridize with $\psi_{1, \overline{\mathrm{M}}}$, lowering the latter's energy, thereby increasing the gap and pushing the energy of $\psi_{2, \overline{\mathrm{M}}}$ up, which detunes it even further from the molecular orbital $\phi_{\mathrm{B}}$ on sublattice B. Thus, initial small differences in the energy level alignment between LUMO+2, on the one hand, and the free-electronlike interface state, on the other hand, together with the associated differences in the charge distribution, lead to an instability at the end of which only one of the sublattices (i.e., A) hybridizes significantly with the free-electron-like interface state, forming a true hybrid interface state. This leads to the strong lateral corrugation of the scattering potential, $U(A)<U(B)$, and in particular to the fact that at sublattice B $U(\vec{r})$ is indeed nearly zero, as found in Fig. 4. Based on this scenario, it is natural that local orbital $\phi_{\mathrm{B}}$ is observed on sublattice B [Fig. 3(e)], at accidental near degeneracy with the hybridized $\psi_{1, \overline{\mathrm{M}}}$ which contains both the free-electron-like interface state and the molecular orbital $\phi_{\mathrm{A}}$.

\section{SUMMARY}

We have applied FD STS, a modified scheme of STS data analysis, to determine the $2 \mathrm{D}$ band structure of the PTCDA/Ag(111) interface state, including its gaps at the 1BZ boundaries of the PTCDA lattice. At its $\bar{\Gamma}$ point the interface state behaves essentially like an upshifted Shockley state. However, the strong scattering by the PTCDA/Ag(111) lattice and the corresponding lateral corrugation potential seen by the interface state show that the 1D model of molecule-metal interface states is oversimplified.

The scattering potential $U(\vec{r})$, reconstructed from the measured band structure, is strongly corrugated. We suggested that this finding indicates a strong influence of the molecular layer on the interface state via the hybridization of their LUMO+2 orbitals. Interestingly, we found that orbitals on only one of the two sublattices (A) are hybridized with the free-electron-like interface state, while orbitals on the other sublattice (B) retain their localized character. The different degrees of hybridization are found to be in agreement with the 
corrugation of the reconstructed $U(\vec{r})$ : Strong hybridization of the type-A molecules leads to the strongly attractive $U(A)$, while the absence of hybridization in the case of the type- $B$ molecules leads to $U(B)$ close to zero.

We close the paper with a final comment on our method to determine band structures from STS data. In the present paper, we have applied FD STS to Bragg scattering within a defect-free adsorbate layer. We note, however, that there is no reason why FD STS could not be applied to impurity scattering in order to observe, e.g., band structures across the complete $1 \mathrm{BZ}$ or even bound states arising from impurity scattering and confinement of dispersive states in 2D.

\section{ACKNOWLEDGMENTS}

We acknowledge fruitful discussions with L. Eschmann, U. Höfer, and M. Rohlfing. F.S.T. acknowledges financial support from the Deutsche Forschungsgemeinschaft through SFB 1083 Structure and Dynamics of Internal Interfaces (Project A 12).
[1] K. Seufert, W. Auwärter, F. J. García De Abajo, D. Ecija, S. Vijayaraghavan, S. Joshi, and J. V. Barth, Nano Lett. 13, 6130 (2013).

[2] M. F. B. Green, T. Esat, C. Wagner, P. Leinen, A. Grötsch, F. S. Tautz, and R. Temirov, Beilstein J. Nanotechnol. 5, 1926 (2014)

[3] K. K. Gomes, W. Mar, W. Ko, F. Guinea, and H. C. Manoharan, Nature (London) 483, 306 (2012).

[4] R. Drost, T. Ojanen, A. Harju, and P. Liljeroth, Nat. Phys. 13, 668 (2017).

[5] M. R. Slot, T. S. Gardenier, P. H. Jacobse, G. C. P. Van Miert, S. N. Kempkes, S. J. M. Zevenhuizen, C. M. Smith, D. Vanmaekelbergh, and I. Swart, Nat. Phys. 13, 672 (2017).

[6] R. Temirov, S. Soubatch, A. Luican, and F. S. Tautz, Nature (London) 444, 350 (2006).

[7] H. Yamane, D. Yoshimura, E. Kawabe, R. Sumii, K. Kanai, Y. Ouchi, N. Ueno, and K. Seki, Phys. Rev. B 76, 165436 (2007)

[8] K. Kanazawa, Y. Sainoo, Y. Konishi, S. Yoshida, A. Taninaka, A. Okada, M. Berthe, N. Kobayashi, O. Takeuchi, and H. Shigekawa, J. Am. Chem. Soc. 129, 740 (2007).

[9] C. H. Schwalb, S. Sachs, M. Marks, A. Schöll, F. Reinert, E. Umbach, and U. Höfer, Phys. Rev. Lett. 101, 146801 (2008).

[10] A. Tamai, A. P. Seitsonen, F. Baumberger, M. Hengsberger, Z. X. Shen, T. Greber, and J. Osterwalder, Phys. Rev. B 77, 075134 (2008).

[11] A. Scheybal, K. Müller, R. Bertschinger, M. Wahl, A. Bendounan, P. Aebi, and T. A. Jung, Phys. Rev. B 79, 115406 (2009).

[12] M. Marks, N. L. Zaitsev, B. Schmidt, C. H. Schwalb, A. Schöll, I. A. Nechaev, P. M. Echenique, E. V. Chulkov, and U. Höfer, Phys. Rev. B 84, 081301(R) (2011).

[13] M. C. E. Galbraith, M. Marks, R. Tonner, and U. Höfer, J. Phys. Chem. Lett. 5, 50 (2014).

[14] M. S. Dyer and M. Persson, New J. Phys. 12, 063014 (2010).

[15] N. Zaitsev, I. Nechaev, and E. Chulkov, J. Exp. Theor. Phys. 110, 114 (2010).

[16] M. Marks, A. Schöll, and U. Höfer, J. Electron. Spectrosc. Relat. Phenom. 195, 263 (2014).

[17] B. W. Heinrich, L. Limot, M. V. Rastei, C. Iacovita, J. P. Bucher, D. M. Djimbi, C. Massobrio, and M. Boero, Phys. Rev. Lett. 107, 216801 (2011).
[18] J. Y. Park, U. D. Ham, S. J. Kahng, Y. Kuk, K. Miyake, K. Hata, and H. Shigekawa, Phys. Rev. B 62, R16341 (2000).

[19] J. Repp, G. Meyer, and K.-H. Rieder, Phys. Rev. Lett. 92, 036803 (2004).

[20] S. M. Hollen, G. A. Gambrel, S. J. Tjung, N. M. Santagata, E. Johnston-Halperin, and J. A. Gupta, Phys. Rev. B 91, 195425 (2015).

[21] H. González-Herrero, P. Pou, J. Lobo-Checa, D. FernándezTorre, F. Craes, A. J. Martínez-Galera, M. M. Ugeda, M. Corso, J. E. Ortega, J. M. Gómez-Rodríguez, R. Pérez, and I. Brihuega, ACS Nano 10, 5131 (2016).

[22] N. Armbrust, F. Schiller, J. Güdde, and U. Höfer, Sci. Rep. 7, 46561 (2017).

[23] S. G. Davison and M. Stȩślicka, Basic Theory of Surface States (Oxford University Press, Oxford, 1992).

[24] F. S. Tautz, Prog. Surf. Sci. 82, 479 (2007).

[25] A. Bendounan, F. Forster, J. Ziroff, F. Schmitt, and F. Reinert, Phys. Rev. B 72, 075407 (2005).

[26] M. Rohlfing, R. Temirov, and F. S. Tautz, Phys. Rev. B 76, 115421 (2007).

[27] L. Petersen, P. T. Sprunger, P. Hofmann, E. Lægsgaard, B. G. Briner, M. Doering, H.-P. Rust, A. M. Bradshaw, F. Besenbacher, and E. W. Plummer, Phys. Rev. B 57, R6858 (1998).

[28] L. Petersen, P. Hofmann, E. W. Plummer, and F. Besenbacher, J. Electron. Spectrosc. Relat. Phenom. 109, 97 (2000).

[29] L. Gross, F. Moresco, L. Savio, A. Gourdon, C. Joachim, and K.-H. Rieder, Phys. Rev. Lett. 93, 056103 (2004).

[30] J. Kröger, L. Limot, H. Jensen, R. Berndt, S. Crampin, and E. Pehlke, Prog. Surf. Sci. 80, 26 (2005).

[31] A. Hauschild, K. Karki, B. C. C. Cowie, M. Rohlfing, F. S. Tautz, and M. Sokolowski, Phys. Rev. Lett. 94, 036106 (2005).

[32] P. Leinen, C. Wagner, R. Temirov, and F. S. Tautz (unpublished).

[33] C. Wagner, M. F. B. Green, P. Leinen, T. Deilmann, P. Krüger, M. Rohlfing, R. Temirov, and F. S. Tautz, Phys. Rev. Lett. 115, 026101 (2015).

[34] P. Hapala, M. Švec, O. Stetsovych, N. J. van Der Heijden, M. Ondráčk, J. van Der Lit, P. Mutombo, I. Swart, and P. Jelínek, Nat. Commun. 7, 11560 (2016).

[35] E. V. Chulkov, V. M. Silkin, and P. M. Echenique, Surf. Sci. 437, 330 (1999).

[36] V. M. Silkin, J. Zhao, F. Guinea, E. V. Chulkov, P. M. Echenique, and H. Petek, Phys. Rev. B 80, 121408(R) (2009). 